\title{
NEW DATA CONCERNING Mn BEARING MINERALS FROM ROŞIA MONTANĂ, METALIFERI MOUNTAINS, ROMANIA
}

\author{
MARCEL BENEA ${ }^{1}$, LAURENT BAILLY ${ }^{2}$, CĂLIN G. TĂMAŞ $^{1}$
}

\begin{abstract}
The paper gives new evidences on some mineral species from Romanian epithermal ore deposits. Microprobe analyses were used to identify the chemical composition of gangue and alteration minerals from Roşia Montană gold deposit. Special attention was given to rhodonite, rhodochrosite, and adularia.
\end{abstract}

Key words: rhodonite, rhodochrosite, Roşia Montană ore deposit, Romania.

\section{INTRODUCTION}

In the course of time the lack of pertinent analytical results gave rise to many controversies regarding Romanian mineralogical references. Many citations of minerals from Romania are based on empirical observations even only macroscopic examinations, sometimes intermingled with more accurate determinations (microscopy, XRD, etc.). As a general rule, the accurate quantitative data (e.g. microprobe results) of many mineral species is still lacking. Several exceptions can be noted: Cook and Damian (1997), Damian (1998), Har (1998), Nicolescu (1998), Damian and Costin (1999), Tămaş and Bailly (1999a). We join to these pioneering works giving new evidences about Mn-bearing minerals (rhodonite, rhodochrosite) and furthermore on K-feldspar (adularia) from Roşia Montană ore deposits.

\section{GENERAL GEOLOGY}

Roşia Montană area belongs to the Bucium Unit as separated by Bleahu et al. (1981) and reconsidered by Balintoni (1994, 1997). The crystalline basement of Baia de Arieş type (Balintoni, 1997) is covered by Cretaceous flysch. Within the so-called "Roşia Montană basin" two volcanic pulses are well developed, while another one is only inferred (Cioflica et al., 1973). First volcanic pulse is an explosive rhyolitic one of Lower Badenian Middle Badenian age (Cioflica et al., 1973). Then follow the main dacite eruptions (upper badenian) - Cetate dacite - which was also followed by a

\footnotetext{
1 "Babeş-Bolyai" University, Faculty of Biology-Geology, Chair of Mineralogy-Petrometallogeny, 1 Kogălniceanu Str., 3400 Cluj-Napoca, Romania

${ }^{2}$ Bureau de Recherches Géologiques et Minières (BRGM), Av. C. Guillemin, B.P. 6009, 45060 Orléans, France
} 
pannonian andesitic phase - Rotunda type (Cioflica et al, 1973, lanovici et al., 1976, Borcoş, 1994).

The high grade and large tonnage Roşia Montană low sulfidation epithermal ore deposit (Mârza et al., 1997, Tămaş and Bailly, 1999b) is related to the dacite activity. We may consign the complex brecciation metallogenetic evolution within the Roşia Montană area as highlighted by Mârza et al. (1997), Tămaş (1998), Tămaş and Bailly (1999b). Consequently, several types of ore body morphologies are well developed: breccias (phreatomagmatic and phreatic types), vein systems (steeply and flatly dipping), stockworks (so-called "volburi"), impregnations as well as placers and paleoplacers (Petrulian, 1934, Ghițulescu and Socolescu, 1940, 1941). The Mn-bearing minerals occur as gangue minerals within breccias, veins and stockwork structures.

\section{PREVIOUS DATA}

Rhodochrosite and rhodonite are the most common minerals among the manganese bearing ones. The misunderstanding regarding the identification of manganese silicate and carbonate lasted for decades. Also, at present, there are a lot of misinterpretations regarding the type locality of these two minerals.

In this respect, according to Papp (1997), the first mention of rhodochrosite is usually attributed to Bergman in 1782 who used the term "magnesium acido aereo mineralisatum" without any description or locality data. Furthermore, Papp (1997) cited a series of authors from 17-th to 18-th centuries who gave evidence on Mn bearing minerals. We will underline below several informations available from Papp (1997). Bergman already described, in 1780, "magnesium aeratum" as the matrix of "minera Nagyayensis" (nagyagite). Rhodochrosite from Săcărâmb (former Nagyag) was described later in details by Fichtel in 1794. His and others' observations were published together with Ruprecht's analytical data on rhodonite from 1783 by Lenz in 1794 . The modern term rhodochrosite was introduced by Hausmann in 1813 who made reference to the first accurate results of the quantitative analysis made by Lampadius in 1800 on a specimen from Kapnik (Cavnic, Romania). The above informations are in agreement with those of Rădulescu and Dimitrescu (1966), and Mureşan et al. (1990), but they do not agreed with those of Udubaşa et al.(1992), Szakall and Gatter (1993) and Nicolescu (1996).

According to the principle of priority (Papp, 1997, p.70) the type locality for rhodochrosite should be Săcărâmb instead of Cavnic.

Refering to rhodonite, the first quantitative analysis was published by Ruprecht (1783, fide Papp, 1997). He analysed the "reddish gangue or 
so-called feldspar" from Kapnik (Cavnic, Romania) known also as "Kapniker Feldspath". He regarded this mineral as a quartz (or jasper) "penetrated" with manganese. However, others like Karsten in 1800 regarded it as a distinct species. The term rhodonite was first used by Jasche in 1819 for a mineral from Elbingerode (Hartz Mts., Germany). According to the principle of priority, the type locality for rhodonite should be Cavnic (Papp, 1997, p.69).

The list of minerals from Roşia Montană Au-Ag epithermal ore deposit comprises about 40 mineral species as cited by Udubasa et al. (1992).

The rhodochrosite from Rosia Montana was mentioned by Cotta and Fellenberg (1862, fide Mike, 1882), Zepharovich (1872), Szabó (1875, fide Papp, 1997), Petrulian (1934), Helke (1938), Ghițulescu and Socolescu (1941), Rădulescu and Dimitrescu (1966). All the available descriptions state that rhodochrosite occurs as globular or reniform masses, with a pinkyellowish colour, forming crusts or filling the veinlets. In most of the cases it is associated with rhodonite. Chalcopyrite, galena as well as gold as granular aggregates within quartz are to be noted. (1938).

The first mention of rhodonite at Roşia Montană was made by Helke

\section{SAMPLING}

The Mn gangue minerals are common within adularia-sericite (low sulfidation) epithermal deposits (Heald et al., 1987, Hedenquist, 1995). The abundance of these minerals within similar type of ore deposits from Romania (e.g. Cavnic) is well known (Mariaş, 1996). Various occurrences of Mnbearing gangue minerals are observed in Roşia Montană ore deposits:

- infill of the open spaces among breccia fragments; it is visible in the open pit (Cetate Breccia) as well as in the underground workings (Racoşi Breccia, +795 level);

- anastomozing veinlets within the stockworks;

- major component of the veins which cut off the dacite, the stockwork structures (Cârnic massif, +853 level), the breccias (Cetate breccia, open pit), as well as within those veins which cut off the Cretaceous flysch (Țarina, Igre mining fields).

In the underground workings, mostly abandoned for decades, the veins rich in $\mathrm{Mn}$ gangue minerals are easily visible even in the huge underground cavities ("coranda") from Cârnic mining field due to their continuity and dark colour. The exposed surface of the veins is black (covered by $\mathrm{Mn}$ oxides) and could be easily traced within the dacite rocks which was substantial whitened due to the widespread potassium silicate and phyllic alterations. At the surface, within the open pit (Cetate open pit), a vein system rich in Mn bearing gangue minerals occurred. This vein system consists of steeply dipping densely parallel vein swarm (sheeted veins) which cut off the so-called Glamm formation (Fig.1). The analysed $\mathrm{Mn}$ bearing minerals samples came from this area (open pit, level +886 ) 
from a vein with an average width of $15 \mathrm{~cm}$. The veins cross over the open pit bench and could be traced on both sides of the access road.

Microscopic studies, both on thin and polished sections were performed. Preliminary ore micros-copy study carried on for several samples from this vein revealed the presence of common sulp-hides (chalcopyrite,

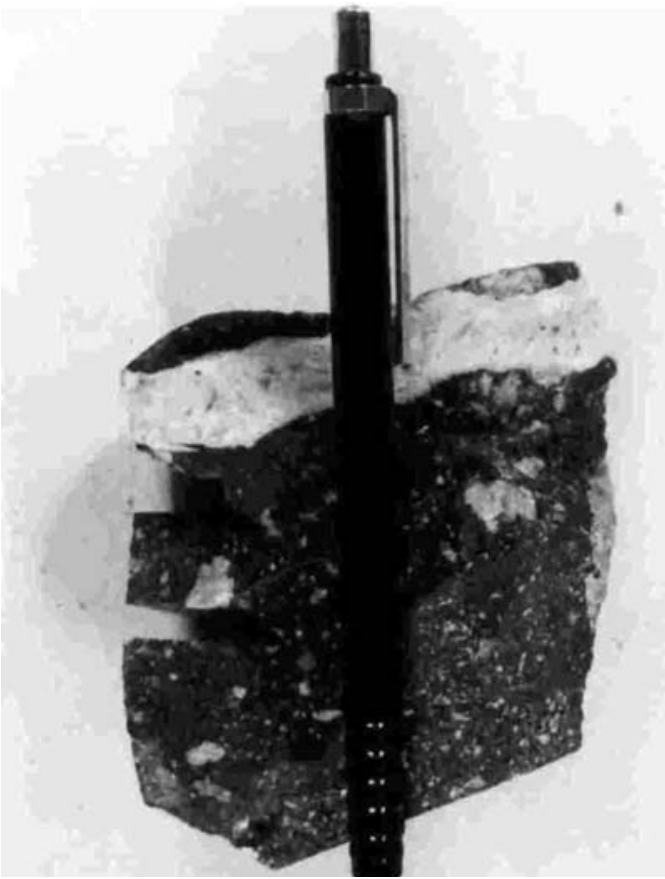

Fig. 1 - Rhodonite - rhodochrosite vein from Glamm formation, Cetate open pit, +886 m level. sphalerite, galena, pyrite) as well as native gold (Tămaş and Bailly, unpubl. data). In thin section, rhodochro-site appears usually as colorless grains, with a medium relief and a perfect rhombohedral cleavage. The other optical properties as birefringence $(0,200-0,210)$ and the negative optic sign complete the observations. On the other side, in some thin sections, rhodonite presents a weak pleochroism, but usually it is also colorless, with a medium relief, good quality cleavage, an inclined extinction $\left(\sim 35^{\circ}\right)$ and low birefringence (0.012-0.014). Rhodonite and rhodochrosite are almost always intimely mixed, rhodochrosite replacing rhodonite.

Fluid inclusion results (Tămaş and Bailly, 1999b) revealed the low salinity $(0.7$ to 4 $w t \% \mathrm{NaCl}$ equiv.) and low homogenization temperatures $\left(200-285^{\circ} \mathrm{C}\right)$ of the fluids. The fluid inclusion analyses were made also on different minerals (adularia, apatite, quartz) related to rhodonite-rhodochrosite deposition across the width of the vein.

\section{ANALYTICAL TECHNIQUES}

The microprobe analyses were performed on similar samples as those subjected for fluid inclusion investigations by Tămaş and Bailly (1999b) (vein fragments from Cetate open pit, level +886) (Fig. 2). 
Fig. 2 - Sketch after a thin polished section on a vein fragment from Cetate open pit

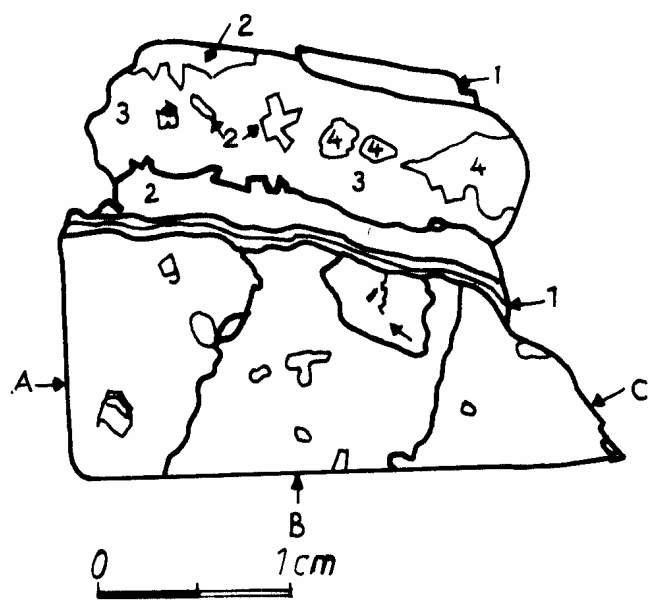
which was subjected to microprobe investigation.

A - fragment of hydrothermalised dacite; $B$ - gray glamm (breccia); the clasts are mainly subangular;

C - black glamm (breccia) poor in clasts;

1 - melange rhodonite - rhodochrosite finely rubanated;

2 - light pink, compact mixing of rhodonite - rhodochrosite;

3 - reddish pink mixing of rhodonite rhodochrosite;

4 - final microcrystalline quartz.

Electron microprobe analyses for this study were performed on rhodonite, rhodochrosite, and adularia, using a SX 50 - CAMECA electron microprobe of B.R.G.M. ${ }^{3}$, Orléans.

A total of 10 elements were analysed: $\mathrm{K}, \mathrm{Si}, \mathrm{Fe}, \mathrm{Ca}, \mathrm{Na}, \mathrm{Ti}, \mathrm{Al}, \mathrm{Mn}$, $\mathrm{Cr}, \mathrm{Mg}$. For all the runs an accelerating voltage of $15 \mathrm{kV}$ was used, with a beam current of $12 \mathrm{nA}$ and a spot size of about $1 \mu \mathrm{m}$. Counting time was 6 $s$ on standards and unknows. A P.A.P. correction program was used for all the analyses.

\section{RESULTS AND DISCUSSION}

A number of 24 analyses on rhodonite, rhodochrosite, and adularia were performed. The selected electron microprobe analyses of these three minerals are listed below. The representative results for the rhodonite are given in Table $1 \mathrm{~A}(\%$ oxides) and Table 1B ( $\%$ elements).

Table 1A

The chemical composition (\% oxides) of rhodonite

\begin{tabular}{|c|r|r|r|r|r|r|r|r|r|}
\hline Point no. & \multicolumn{1}{c|}{3} & \multicolumn{1}{c|}{4} & \multicolumn{1}{c|}{12} & \multicolumn{1}{c|}{13} & \multicolumn{1}{c|}{14} & \multicolumn{1}{c|}{15} & 16 & 18 & \multicolumn{1}{c|}{19} \\
\hline $\mathrm{K}_{2} \mathrm{O}$ & 0.018 & 0.034 & 0.033 & 0.024 & 0.118 & 0.018 & 0.010 & 0.031 & 0.069 \\
\hline $\mathrm{SiO}_{2}$ & 44.628 & 44.574 & 44.497 & 44.491 & 44.452 & 45.376 & 43.684 & 44.38 & 45.394 \\
\hline $\mathrm{FeO}$ & 0.293 & 0.256 & 0.244 & 0.124 & 0.084 & 0.172 & 0.050 & 0.169 & 0.018 \\
\hline $\mathrm{CaO}$ & 4.732 & 6.394 & 3.217 & 4.371 & 4.151 & 3.292 & 2.635 & 2.637 & 4.871 \\
\hline $\mathrm{Na}_{2} \mathrm{O}$ & 0.019 & 0.130 & 0.024 & 0.015 & 0.446 & 0.078 & 0.000 & 0.012 & 0.097 \\
\hline $\mathrm{TiO}_{2}$ & 0.000 & 0.060 & 0.010 & 0.088 & 0.058 & 0.000 & 0.055 & 0.000 & 0.000 \\
\hline $\mathrm{Al}_{2} \mathrm{O}_{3}$ & 0.326 & 0.257 & 0.393 & 0.264 & 0.183 & 0.367 & 0.163 & 0.326 & 0.416 \\
\hline $\mathrm{MnO}$ & 49.835 & 47.457 & 50.289 & 49.195 & 48.625 & 49.081 & 51.774 & 50.076 & 49.553 \\
\hline $\mathrm{MgO}$ & 0.549 & 0.590 & 0.623 & 0.413 & 0.546 & 0.904 & 0.554 & 0.701 & 0.360 \\
\hline & 100.400 & 99.752 & 99.330 & 98.985 & 98.663 & 99.288 & 98.925 & 98.332 & 100.778 \\
\hline
\end{tabular}

${ }^{3}$ Analyst Ch. Gilles, BRGM, Orléans, France 
MARCEL BENEA, LAURENT BAILLY, CĂLIN G. TĂMAŞ

\begin{tabular}{|l|r|r|r|r|r|r|r|r|}
\hline Point no. & \multicolumn{1}{c|}{20} & \multicolumn{1}{c|}{22} & \multicolumn{1}{c|}{23} & \multicolumn{1}{c|}{29} & \multicolumn{1}{c|}{33} & \multicolumn{1}{c|}{34} & \multicolumn{1}{c|}{35} & \multicolumn{1}{c|}{37} \\
\hline $\mathrm{K}_{2} \mathrm{O}$ & 0.027 & 0.122 & 0.039 & 0.071 & 0.007 & 0.148 & 0.000 & 0.000 \\
\hline $\mathrm{SiO}_{2}$ & 45.926 & 56.644 & 61.466 & 56.407 & 45.212 & 67.999 & 45.582 & 46.908 \\
\hline $\mathrm{FeO}$ & 0.121 & 0.105 & 0.087 & 0.162 & 0.071 & 0.018 & 0.005 & 0.144 \\
\hline $\mathrm{CaO}$ & 3.936 & 2.902 & 2.742 & 3.075 & 4.332 & 3.908 & 4.500 & 9.041 \\
\hline $\mathrm{Na}_{2} \mathrm{O}$ & 0.000 & 0.120 & 0.082 & 0.171 & 0.034 & 0.148 & 0.175 & 0.051 \\
\hline $\mathrm{TiO}_{2}$ & 0.010 & 0.000 & 0.000 & 0.013 & 0.000 & 0.007 & 0.023 & 0.047 \\
\hline $\mathrm{Al}_{2} \mathrm{O}_{3}$ & 0.308 & 0.326 & 0.326 & 0.347 & 0.612 & 0.465 & 0.565 & 0.238 \\
\hline $\mathrm{MnO}$ & 49.540 & 37.790 & 33.193 & 38.814 & 50.081 & 28.285 & 50.017 & 43.787 \\
\hline $\mathrm{MgO}$ & 0.657 & 0.532 & 0.376 & 0.509 & 0.466 & 0.229 & 0.400 & 0.257 \\
\hline & 100.525 & 98.541 & 98.311 & 99.569 & 100.815 & 101.207 & 101.267 & 100.473 \\
\hline
\end{tabular}

Table 1B

The chemical composition (\% elements) of rhodonite

\begin{tabular}{|c|c|c|c|c|c|c|c|c|c|}
\hline Point no. & 3 & 4 & 12 & 13 & 14 & 15 & 16 & 18 & 19 \\
\hline $\mathrm{K}$ & 0.001 & 0.001 & 0.001 & 0.001 & 0.003 & 0.001 & 0.000 & 0.001 & 0.002 \\
\hline $\mathrm{Si}$ & 0.969 & 0.969 & 0.975 & 0.977 & 0.977 & 0.985 & 0.968 & 0.981 & 0.977 \\
\hline $\mathrm{Fe}$ & 0.005 & 0.005 & 0.004 & 0.002 & 0.002 & 0.003 & 0.001 & 0.003 & 0.000 \\
\hline $\mathrm{Ca}$ & 0.110 & 0.149 & 0.076 & 0.103 & 0.098 & 0.077 & 0.063 & 0.062 & 0.112 \\
\hline $\mathrm{Na}$ & 0.001 & 0.006 & 0.001 & 0.001 & 0.019 & 0.003 & 0.000 & 0.001 & 0.004 \\
\hline $\mathrm{Ti}$ & 0.000 & 0.001 & 0.000 & 0.002 & 0.001 & 0.000 & 0.001 & 0.000 & 0.000 \\
\hline $\mathrm{Al}$ & 0.008 & 0.007 & 0.010 & 0.007 & 0.005 & 0.009 & 0.004 & 0.009 & 0.011 \\
\hline $\mathrm{Mn}$ & 0.916 & 0.873 & 0.933 & 0.915 & 0.906 & 0.903 & 0.972 & 0.937 & 0.903 \\
\hline $\mathrm{Mg}$ & 0.018 & 0.019 & 0.020 & 0.014 & 0.018 & 0.029 & 0.018 & 0.023 & 0.012 \\
\hline & 2.028 & 2.029 & 2.021 & 2.019 & 2.028 & 2.010 & 2.027 & 2.016 & 2.021 \\
\hline
\end{tabular}

\begin{tabular}{|c|c|c|c|c|c|c|c|c|}
\hline Point no. & 20 & 22 & 23 & 29 & 33 & 34 & 35 & 37 \\
\hline $\mathrm{K}$ & 0.001 & 0.003 & 0.001 & 0.002 & 0.000 & 0.003 & 0.000 & 0.000 \\
\hline $\mathrm{Si}$ & 0.986 & 1.132 & 1.187 & 1.123 & 0.973 & 1.232 & 0.975 & 0.994 \\
\hline $\mathrm{Fe}$ & 0.002 & 0.002 & 0.001 & 0.003 & 0.001 & 0.000 & 0.000 & 0.003 \\
\hline $\mathrm{Ca}$ & 0.091 & 0.062 & 0.057 & 0.066 & 0.100 & 0.076 & 0.103 & 0.205 \\
\hline $\mathrm{Na}$ & 0.000 & 0.005 & 0.003 & 0.007 & 0.001 & 0.005 & 0.007 & 0.002 \\
\hline $\mathrm{Ti}$ & 0.000 & 0.000 & 0.000 & 0.000 & 0.000 & 0.000 & 0.000 & 0.001 \\
\hline $\mathrm{Al}$ & 0.008 & 0.008 & 0.007 & 0.008 & 0.016 & 0.010 & 0.014 & 0.006 \\
\hline $\mathrm{Mn}$ & 0.901 & 0.640 & 0.543 & 0.654 & 0.913 & 0.434 & 0.906 & 0.786 \\
\hline $\mathrm{Mg}$ & 0.021 & 0.016 & 0.011 & 0.015 & 0.015 & 0.006 & 0.013 & 0.008 \\
\hline & 2.010 & 1.868 & 1.811 & 1.877 & 2.020 & 1.767 & 2.018 & 2.004 \\
\hline
\end{tabular}

The representative electron microprobe analyses for rhodochrosite (6 runs) are listed in Table 2A (\% oxides) and in Table 2B (\% elements).

Besides rhodonite, the thin section study revealed also the presence of plagioclase and adularia. The electron microprobe analysis of adularia (\% oxides) is given in Table 3 . 
NEW DATA CONCERNING Mn BEARING MINERALS FROM ROŞIA MONTANĂ

Table 2 A

The chemical composition (\% oxides) of rhodochrosite

\begin{tabular}{|l|r|r|r|r|r|r|}
\hline Point no. & \multicolumn{1}{|c|}{6} & \multicolumn{1}{c|}{8} & \multicolumn{1}{c|}{10} & \multicolumn{1}{c|}{11} & \multicolumn{1}{c|}{21} & \multicolumn{1}{c|}{31} \\
\hline $\mathrm{K}_{2} \mathrm{O}$ & 0.027 & 0.000 & 0.000 & 0.000 & 0.019 & 0.000 \\
\hline $\mathrm{SiO}$ & 0.439 & 0.049 & 0.071 & 0.056 & 0.122 & 9.920 \\
\hline $\mathrm{FeO}$ & 0.000 & 0.062 & 0.242 & 0.140 & 0.015 & 0.068 \\
\hline $\mathrm{CaO}$ & 2.574 & 9.591 & 5.956 & 8.012 & 2.345 & 3.270 \\
\hline $\mathrm{Na}_{2} \mathrm{O}$ & 0.039 & 0.008 & 0.044 & 0.043 & 0.088 & 0.071 \\
\hline $\mathrm{TiO}_{2}$ & 0.008 & 0.038 & 0.013 & 0.000 & 0.020 & 0.035 \\
\hline $\mathrm{Al}_{2} \mathrm{O}_{3}$ & 0.000 & 0.017 & 0.009 & 0.021 & 0.023 & 0.079 \\
\hline $\mathrm{MnO}$ & 57.688 & 47.836 & 53.797 & 49.374 & 57.612 & 55.628 \\
\hline $\mathrm{MgO}$ & 0.348 & 0.617 & 0.809 & 0.730 & 0.464 & 0.280 \\
\hline & 61.123 & 58.218 & 60.941 & 58.376 & 60.708 & 69.351 \\
\hline
\end{tabular}

Table 2B

The chemical composition (\% elements) of rhodochrosite

\begin{tabular}{|l|c|c|c|c|c|c|}
\hline Point no. & 6 & 8 & 10 & 11 & 21 & 31 \\
\hline $\mathrm{K}$ & 0.001 & 0.000 & 0.000 & 0.000 & 0.001 & 0.000 \\
\hline $\mathrm{Si}$ & 0.017 & 0.002 & 0.003 & 0.002 & 0.005 & 0.310 \\
\hline $\mathrm{Fe}$ & 0.000 & 0.002 & 0.008 & 0.005 & 0.001 & 0.002 \\
\hline $\mathrm{Ca}$ & 0.104 & 0.396 & 0.238 & 0.332 & 0.096 & 0.110 \\
\hline $\mathrm{Na}$ & 0.003 & 0.001 & 0.003 & 0.003 & 0.007 & 0.004 \\
\hline $\mathrm{Ti}$ & 0.000 & 0.001 & 0.000 & 0.000 & 0.001 & 0.001 \\
\hline $\mathrm{Al}$ & 0.000 & 0.001 & 0.000 & 0.001 & 0.001 & 0.003 \\
\hline $\mathrm{Mn}$ & 1.847 & 1.561 & 1.702 & 1.615 & 1.863 & 1.474 \\
\hline $\mathrm{Mg}$ & 0.020 & 0.035 & 0.045 & 0.042 & 0.026 & 0.013 \\
\hline & 1.992 & 1.999 & 1.999 & 1.999 & 1.999 & 1.917 \\
\hline
\end{tabular}

Table 3

The chemical composition of adularia and the resulted mineral \% $(\mathrm{O}=8)$

\begin{tabular}{|c|c|c|c|c|}
\hline Point no. & 5 & & 5 & Mineral $\%$ and calculated formula \\
\hline $\mathrm{K}_{2} \mathrm{O}$ & 16.47 & $\mathrm{~K}$ & 0.97 & \multirow{10}{*}{$\begin{array}{l}\text { Adularia } 97.36 \% \\
\text { Albite } 1.63 \% \\
\text { Quartz } 0.51 \% \\
\text { Pyroxmangite } 0.37 \% \\
\text { Rhodonite } 0.06 \%\end{array}$} \\
\hline $\mathrm{SiO}_{2}$ & 64.81 & $\mathrm{Si}$ & 3.00 & \\
\hline $\mathrm{FeO}$ & 0.13 & $\mathrm{Fe}$ & 0.01 & \\
\hline $\mathrm{CaO}$ & 0.00 & $\mathrm{Ca}$ & 0.00 & \\
\hline $\mathrm{Na}_{2} \mathrm{O}$ & 0.19 & $\mathrm{Na}$ & 0.02 & \\
\hline $\mathrm{TiO}_{2}$ & 0.00 & $\mathrm{Ti}$ & 0.00 & \\
\hline $\mathrm{Al}_{2} \mathrm{O}_{3}$ & 18.15 & $\mathrm{Al}$ & 0.99 & \\
\hline $\mathrm{MnO}$ & 0.17 & $\mathrm{Mn}$ & 0.01 & \\
\hline \multirow[t]{2}{*}{$\mathrm{MgO}$} & 0.00 & $\mathrm{Mg}$ & 0.00 & \\
\hline & 99.93 & & 5.00 & \\
\hline
\end{tabular}


The mineralogical calculations made for the runs focused on rhodonite areas are listed in the table below (Table 4).

Table 4

The mineral $\%$ on the rhodonite areas and the calculated formula

\begin{tabular}{|c|c|c|c|c|c|}
\hline \multirow{2}{*}{$\begin{array}{l}\text { Point } \\
\text { no. }\end{array}$} & \multicolumn{4}{|c|}{ Mineral \% } & \multirow[b]{2}{*}{ Rhodonite } \\
\hline & Rhodonite & $\begin{array}{c}\text { Adulari } \\
a\end{array}$ & $\begin{array}{c}\text { Albit } \\
e\end{array}$ & Anorthite & \\
\hline 3 & 99.69 & 0.11 & 0.16 & 0.44 & $\begin{array}{c}\left(\mathrm{Mn}_{0.916}, \mathrm{Ca}_{0.110}, \mathrm{Fe}_{0.005}\right. \\
\left.\mathrm{Mg}_{0.018}\right)\left[\mathrm{Si}_{0.969} \mathrm{O}_{3}\right]\end{array}$ \\
\hline 4 & 98.39 & 0.22 & 1.10 & - & $\begin{array}{c}\left(\mathrm{Mn}_{0.873}, \mathrm{Ca}_{0.149}, \mathrm{Fe}_{0.005}\right. \\
\left.\mathrm{Mg}_{0.019}\right)\left[\mathrm{Si}_{0.969} \mathrm{O}_{3}\right]\end{array}$ \\
\hline 12 & 98.40 & 0.22 & 0.20 & 0.49 & $\begin{array}{c}\left(\mathrm{Mn}_{0.933,}, \mathrm{Ca}_{0.075}, \mathrm{Fe}_{0.004}\right. \\
\left.\mathrm{Mg}_{0.020}\right)\left[\mathrm{Si}_{0.975} \mathrm{O}_{3}\right] \\
\end{array}$ \\
\hline 13 & 98.36 & 0.16 & 0.11 & 0.27 & $\begin{array}{c}\left(\mathrm{Mn}_{0.915}, \mathrm{Ca}_{0.103}, \mathrm{Fe}_{0.002}\right. \\
\left.\mathrm{Mg}_{0.014}\right)\left[\mathrm{Si}_{0.977} \mathrm{O}_{3}\right]\end{array}$ \\
\hline 14 & 97.21 & 0.66 & 0.32 & - & $\begin{array}{c}\left(\mathrm{Mn}_{0.906}, \mathrm{Ca}_{0.098}, \mathrm{Fe}_{0.002}\right. \\
\left.\mathrm{Mg}_{0.018}\right)\left[\mathrm{Si}_{0.977} \mathrm{O}_{3}\right]\end{array}$ \\
\hline 15 & 98.15 & 0.11 & 0.68 & 0.33 & $\begin{array}{c}\left(\mathrm{Mn}_{0.903,} \mathrm{Ca}_{0.077}, \mathrm{Fe}_{0.003}\right. \\
\left.\mathrm{Mg}_{0.029}\right)\left[\mathrm{Si}_{0.985} \mathrm{O}_{3}\right] \\
\end{array}$ \\
\hline 16 & 98.57 & 0.06 & - & 0.24 & $\begin{array}{c}\left(\mathrm{Mn}_{0.972,} \mathrm{Ca}_{0.063}, \mathrm{Fe}_{0.001}\right. \\
\left.\mathrm{Mg}_{0.018}\right)\left[\mathrm{Si}_{0.968} \mathrm{O}_{3}\right]\end{array}$ \\
\hline 18 & 97.62 & 0.17 & 0.10 & 0.44 & $\begin{array}{c}\left(\mathrm{Mn}_{0.937}, \mathrm{Ca}_{0.062}, \mathrm{Fe}_{0.003}\right. \\
\left.\mathrm{Mg}_{0.023}\right)\left[\mathrm{Si}_{0.981} \mathrm{O}_{3}\right]\end{array}$ \\
\hline 19 & 99.26 & 0.39 & 0.84 & 0.28 & $\begin{array}{c}\left(\mathrm{Mn}_{0.903}, \mathrm{Ca}_{0.112}\right. \\
\left.\mathrm{Mg}_{0.012}\right)\left[\mathrm{Si}_{0.977} \mathrm{O}_{3}\right]\end{array}$ \\
\hline 20 & 99.90 & 0.17 & - & 0.44 & $\begin{array}{c}\left(\mathrm{Mn}_{0.901}, \mathrm{Ca}_{0.091}, \mathrm{Fe}_{0.002}\right. \\
\left.\mathrm{Mg}_{0.021}\right)\left[\mathrm{Si}_{0.986} \mathrm{O}_{3}\right]\end{array}$ \\
\hline 22 & 96.82 & 0.72 & 1.00 & - & $\begin{array}{c}\left(\mathrm{Mn}_{0.640}, \mathrm{Ca}_{0.062}, \mathrm{Fe}_{0.002}\right. \\
\left.\mathrm{Mg}_{0.016}\right)\left[\mathrm{Si}_{1.132} \mathrm{O}_{3}\right]\end{array}$ \\
\hline 23 & 97.16 & 0.23 & 0.68 & 0.24 & $\begin{array}{c}\left(\mathrm{Mn}_{0.543,}, \mathrm{Ca}_{0.057}, \mathrm{Fe}_{0.001}\right. \\
\left.\mathrm{Mg}_{0.011}\right)\left[\mathrm{Si}_{1.187} \mathrm{O}_{3}\right]\end{array}$ \\
\hline 29 & 97.74 & 0.40 & 1.42 & - & $\begin{array}{c}\left(\mathrm{Mn}_{0.654}, \mathrm{Ca}_{0.066}, \mathrm{Fe}_{0.003}\right. \\
\left.\mathrm{Mg}_{0.015}\right)\left[\mathrm{Si}_{1.123} \mathrm{O}_{3}\right]\end{array}$ \\
\hline 33 & 99.63 & 0.06 & 0.26 & 0.87 & $\begin{array}{c}\left(\mathrm{Mn}_{0.913}, \mathrm{Ca}_{0.100}, \mathrm{Fe}_{0.001}\right. \\
\left.\mathrm{Mg}_{0.015}\right)\left[\mathrm{Si}_{0.973} \mathrm{O}_{3}\right]\end{array}$ \\
\hline 34 & 98.97 & 0.89 & 1.26 & 0.09 & $\begin{array}{c}\left(\mathrm{Mn}_{0.434}, \mathrm{Ca}_{0.076}\right. \\
\left.\mathrm{Mg}_{0.006}\right)\left[\mathrm{Si}_{1.232} \mathrm{O}_{3}\right]\end{array}$ \\
\hline 35 & 99.34 & - & 1.47 & 0.44 & $\begin{array}{c}\left(\mathrm{Mn}_{0.906}, \mathrm{Ca}_{0.103}\right. \\
\left.\mathrm{Mg}_{0.013}\right)\left[\mathrm{Si}_{0.975} \mathrm{O}_{3}\right]\end{array}$ \\
\hline 37 & 99.76 & - & 0.42 & 0.24 & $\begin{array}{c}\left(\mathrm{Mn}_{0.786,} \mathrm{Ca}_{0.205}, \mathrm{Fe}_{0.003}\right. \\
\left.\mathrm{Mg}_{0.008}\right)\left[\mathrm{Si}_{0.994} \mathrm{O}_{3}\right]\end{array}$ \\
\hline
\end{tabular}

The final formula of the composition of rhodonite $\left(\mathrm{Mn}_{0.826}, \mathrm{Ca}_{0.095}\right.$, $\left.\mathrm{Fe}_{0.002}, \mathrm{Mg}_{0.016}\right)\left[\mathrm{Si}_{1.022} \mathrm{O}_{3}\right]$ was obtained as a mean value of the coefficients for each chemical element. 
The results of mineralogical calculations made for rhodochrosite are given in table below (Table 5).

Table 5

The calculated formula for rhodochrosite

\begin{tabular}{|c|c|}
\hline Point no. & Rhodochrosite \\
\hline 6 & $\left(\mathrm{Mn}_{1.847}, \mathrm{Ca}_{0.104}, \mathrm{Mg}_{0.020}\right)\left[\mathrm{C}_{0.990} \mathrm{O}_{3}\right]$ \\
\hline 8 & $\left(\mathrm{Mn}_{1.561}, \mathrm{Ca}_{0.396}, \mathrm{Mg}_{0.035}\right)\left[\mathrm{C}_{0.978} \mathrm{O}_{3}\right]$ \\
\hline 10 & $\left(\mathrm{Mn}_{1.702}, \mathrm{Ca}_{0.238}, \mathrm{Mg}_{0.045}\right)\left[\mathrm{C}_{0.974} \mathrm{O}_{3}\right]$ \\
\hline 11 & $\left(\mathrm{Mn}_{1.615}, \mathrm{Ca}_{0.332}, \mathrm{Mg}_{0.042}\right)\left[\mathrm{C}_{0.975} \mathrm{O}_{3}\right]$ \\
\hline 21 & $\left(\mathrm{Mn}_{1.863}, \mathrm{Ca}_{0.096}, \mathrm{Mg}_{0.026}\right)\left[\mathrm{C}_{0.986} \mathrm{O}_{3}\right]$ \\
\hline 31 & $\left(\mathrm{Mn}_{1.474}, \mathrm{Ca}_{0.110}, \mathrm{Mg}_{0.013}\right)\left[\mathrm{C}_{0.991} \mathrm{O}_{3}\right]$ \\
\hline
\end{tabular}

The final expression of the composition of rhodochrosite $\left(\mathrm{Mn}_{1.677}\right.$, $\left.\mathrm{Ca}_{0.213}, \mathrm{Mg}_{0.030}\right)\left[\mathrm{Ca}_{0.982} \mathrm{O}_{3}\right]$ was obtained using the mean values of the coefficients for each element.

\section{ACKNOWLEDGEMENTS}

We highly appreciate the technical and financial support offered by B.R.G.M., Orléans, France.

\section{REFERENCES}

1. Balintoni, I. (1994) - Structure of the Apuseni Mountains. ALCAPA II, Geological evolution of the Alpin - Carpathian - Pannonian System. Field Guidebook, South Carpathians and Apuseni Mountains. Rom. Journ. Tect. and Reg. Geol., v. 75, Suppl. no. 2, p. 51-58, Bucureşti

2. Balintoni, I. (1997) - Geotectonica terenurilor metamorfice din Romania. Ed. Carpatica, 176 p., Cluj-Napoca

3. Bleahu, M., Lupu, M., Patrulius, S., Bordea, S., Ştefan, A., Panin, S. (1981) The structure of the Apuseni Mountains. Carp. Balk. Geol. Assoc. XII Congr., Guide to Excursion B3, Inst. Geol. Geophys., Bucharest

4. Borcoş, M. (1994) - Volcanicity/Metallogeny in the South Apuseni Mountains (Metaliferi Mountains), in Borcos, M., and Vlad, S. (eds.): Field trip guide - Plate tectonics and metallogeny in the East Carpathians and Apuseni Mts., june 7-19, p. 32-41, IGCP Project 356

5. Cioflica, G., Savu, H., Borcoş, M., Ştefan, A., Istrate, G. (1973) - Alpine volcanism and metallogenesis in the Apuseni Mountains. Symposium Volcanism and Metallogenesis, Bucharest, 1973. Guide to Excursion 3AB, Guidebook series, no., 13, 70p.

6. Cook, N.J., Damian, G.Ş. (1997) - New data on "Plumosite" and other sulphosalt minerals from Herja hydrothermal ore deposit, Baia Mare district, Rumania. Geologica Carpatica, No. 48, v. 6, p. 387-399 
7. Damian, Floarea (1998) - Studiul mineralogic, geochimic şi genetic al mineralizațiilor de sulfuri din regiunea Nistru - Baia Mare. Teză de doctorat, Universitatea din Bucureşti, Fac. Geol. Geofiz, 117 p.

8. Damian, G., Costin, D. (1999) - Tetrahedrite - bournonite in Breiner - Băiuț mineralization (Gutâi Mts., Eastern Carpathians). Studia Univ. Babeş-Bolyai, Geologia, XLIV, 1, p. 137-149

9. Fleischer, M. (1987) - Glossary of Mineral Species. $5^{\text {th }}$ edition, Mineralogical Record, Tucson, Arizona.

10.Ghițulescu T.P., Socolescu M. (1940) - Les gisements sedimentaires d'or d'age tertiaire dans les Monts Apuseni. C.R., t. XXIII, (1934-1935), p. 37-46, Bucureşti

11.Ghițulescu T.P., Socolescu M. (1941) - Étude geologique et miniere des Monts Metaliferes (Quadrilatere aurifere et regions environnantes). An. Inst. Geol. Rom., vol.XXI, p.181-465, Bucureşti

12.Har, N. (1998) - Cercetări petrografice asupra rocilor bazaltice neogencuaternare din Munții Apuseni. Teză de doctorat, Univ. Babeş-Bolyai, ClujNapoca, 308 p. (unpubl.)

13. Heald, P., Foley, N. K., Hayba, D. O. (1987) - Comparative anatomy of volcanic-hosted epithermal deposits: acide-sulfate and adularia-sericite types. Econ. Geol., v. 82, No. 1, p. 1-26

14. Hedenquist, J. W. (1995) - Origin of and exploration for epithermal gold deposits. Budapest, $121 \mathrm{p}$.

15. Helke, A. (1938) - Die jungvulkanischen Gold- und Silber-Erzlagerstätten des Karpatenbogens. Archiv Lagerstättenforsch, 66.

16.Ianovici, V., Borcoş, M., Bleahu, M., Patrulius, D., Lupu, M., Dimitrescu, R., Savu, H. (1976) - Geologia Munților Apuseni. Ed. Acad. Bucureşti, 631 p.

17.Mariaş, Z. Fr. (1996) - Câmpul metalogenetic Cavnic, caracterizare geostructurală şi petrometalogenetică. Teză de doctorat, Univ. Babeş-Bolyai, Cluj-Napoca, 224 p. (unpubl.)

18.Mârza, I., Tămaş, C., Ghergari, L. (1997) - Low sulfidation epithermal gold deposits from Roşia Montană, Metaliferi Mountains, Romania. St. Cerc. Geol., t. 42, p. 3-12, Bucureşti

19.Mike, T. (1882) - Magyarország Ásványai. Budapest, 565 p.

20.Mureşan, I., Tîrlea-Hoțiu, lulia, Cîmpean, Alexandrina (1990) - Minerale din România descrise prima dată în literatura mondială (considerații generale). Studia Univ. Babeş-Bolyai, Ser. Geol., 25, p. 3-33, Cluj-Napoca.

21.Nickel, E.H., Nichols - M.C. (1991), Mineral Reference Manual. Van Nostrand Reinhold, New York, $250 \mathrm{p}$.

22. Nicolescu, Şt. (1996) - List of valid and discredited mineral species and varieties first described from Romania, in Nicolescu Şt. (ed.), Excursion Guide. Field Trip E1. Banat and Transylvanian Gold District, Romania, p. 37-43. Budapest, Magyarhoni Földtani Társulat.

23. Nicolescu, Şt. (1998) - Skarn genesis at Ocna de Fier - Dognecea, south-west Romania; Earth Sciences Centre, Goteborg University, A36, Goteborg.

24.Papp, G. (1997) - On the mineral species first described from the Carpathian region. Acta Min.-Petr., XXXVIII, Suppl., p. 65-75, Szeged. 
25.Petrulian, N. (1934) - Étude calcographique du gisement aurifere Roşia Montană, (Transylvanie, Roumanie), An. Inst. Geol. Rom., vol XVI, p. 499-538, Bucureşti.

26.Rădulescu, D., Dimitrescu, R. (1966) - Mineralogia topografică a României. Ed. Acad. Române, Bucureşti, 376 p.

27.Szakall, S., Gatter, I. (1993) - Magyarországi Ásványfajok. Fair System Kft., Miskolc.

28.Tămaş, C.G. (1998) - Descriptive breccia types in the Roşia Montana low sulphidation epithermal ore deposit, Apuseni Mountains, Romania. Studia Univ. Babeş-Bolyai, Geologia, XLIII, 1, p. 75-88

29.Tămaş, C.G., Bailly, L. (1999a) -Microprobe data regarding the bournonite $\mathrm{PbCuSbS}_{3}$ from Baia de Arieş, Apuseni Mts., Romania. Studia Univ. "BabeşBolyai", Geologia, XLIV, 1, p. 23-30

30.Tămaş, C.G., Bailly, L. (1999b) - Roşia Montană epithermal ore deposit evidence from fluid inclusion study. Studia Univ. "Babeş-Bolyai", Geologia, XLIV, 1, p. 49-56

31.Udubaşa, Gh., llinca, Gh., Marincea, St., Săbău, G., Rădan, S. (1992) Minerals in Romania: the State of the Art 1991. Rom. J. Mineralogy, 75, 1-51, Bucureşti.

32.Zepharovich, V.v. (1872) - Mineralogisches Lexicon des Kaiserthums Österreich. Band 2, Wien. 